Review began 10/25/2021 Review ended 11/14/2021 Published 11/17/2021

๑) Copyright 2021

Huffman et al. This is an open access article distributed under the terms of the Creative Commons Attribution License CCBY 4.0., which permits unrestricted use, distribution, and reproduction in any medium, provided the original author and source are credited.

\section{An Internal Review of Rates of Palliative Medicine Referral for Patients With Advanced Pancreatic Cancer}

Deanna L. Huffman ${ }^{1}$, Urwat T. Vusqa ${ }^{1}$, Karthik Shankar ${ }^{1}$, Lynna Alnimer ${ }^{2}$, Yazan Samhouri ${ }^{3}$, Srividya Srinivasamaharaj ${ }^{4}$, Srikrishna V. Malayala ${ }^{5}$, Dulabh Monga ${ }^{6}$

1. Internal Medicine, Allegheny Health Network, Pittsburgh, USA 2. Internal Medicine, Ascension Providence Hospital, Southfield, USA 3. Department of Hematology and Cellular Therapy, Allegheny Health Network Cancer Institute, Pittsburgh, USA 4. Hematology, Medical Oncology, Piedmont Healthcare, Columbus, USA 5. Internal Medicine, Temple University Hospital, Philadelphia, USA 6. Department of Hematology and Oncology, Allegheny Health Network Cancer Institute, Pittsburgh, USA

Corresponding author: Urwat T. Vusqa, vusqa.urwat@ahn.org

\section{Abstract \\ Background}

The American Society of Clinical Oncology recommends that patients with advanced cancer receive palliative care services in concurrence with active treatment. While the benefits of palliative care are clear, integration of palliative care can be challenging. We aim to review rates of palliative care consultation in patients with advanced pancreatic cancer at our institution, intending to improve these rates.

\section{Methods}

We retrospectively reviewed the electronic records of all patients with pancreatic cancer treated at Allegheny General Hospital diagnosed between 2009-2020. Summary statistics are presented as percentages for categorical data and median with interquartile range for quantitative data.

\section{Results}

Of the 171 patients reviewed, 121 completed all treatment and evaluation within our health network (Pittsburgh, United States). The median age was 63 years (IQR 40-91 years); 55 patients (45\%) were male; the majority were white (107 patients, $88 \%$ ). At the time of diagnosis, $28 \%$ of our patients had stage IV disease (34 patients), and $19.8 \%$ of patients who developed stage IV disease had palliative care referrals.

\section{Conclusions}

Palliative care is an integral part of usual care for advanced pancreatic cancer. Our analysis showed that palliative care is underutilized in our hospital. We aim to improve palliative care integration in our patients' care by adding a hard stop to electronic medical records to remind physicians to offer palliative care to our patients with pancreatic cancer and to arrange lecture series to emphasize the importance of palliative care in this setting.

Categories: Pain Management, Oncology, Palliative Care

Keywords: electronic health record, quality review, pain management, palliative care, pancreatic cancer

\section{Introduction}

Pancreatic cancer is the fourth leading cause of cancer-related death in the developed world and seventh worldwide [1]. It is associated with high mortality, with median survival of 4.6 months and overall survival of $3 \%$ at five years [2-3]. The poor outcomes are attributed to non-specific symptoms, leading to a delay in diagnosis [1-3]. Moreover, it is associated with aggressive tumor biology and early spread [1-3]. The treatment of pancreatic cancer includes radical resection of the pancreas with adjuvant chemotherapy [1]. However, only $20 \%$ of those initially diagnosed are eligible for the definitive treatment, as the remaining $80 \%$ present with advanced disease [1-3]. Even with complete resection, the prognosis remains grim; for node-negative disease, the five-year survival after pancreaticoduodenectomy is $\sim 25-30 \%$ and for nodepositive disease, the five-year survival drops to $10 \%$ [1-4].

As pancreatic cancer is usually diagnosed at an advanced stage, patients often suffer from a high symptom burden and associated emotional stressors. Bothersome symptoms can be wide-ranging and include pain, jaundice with associated pruritus, ascites, pancreatic exocrine insufficiency leading to digestive difficulty, pancreatic endocrine insufficiency leading to diabetes, gastric outlet obstruction, anorexia, and weight loss [5-6]. The diagnosis of pancreatic cancer is also associated with the development of anxiety, depression, and sleep disturbances, and patients diagnosed with pancreatic cancer are more likely to commit suicide than the 


\section{Cureus}

general population [7-9].

The integration of palliative care services into the treatment of pancreatic cancer can help patients reduce pain, improve quality of life, and has even been shown to prolong survival [4-5]. Several randomized controlled trials have been completed in the past decade, which confirm these benefits, and the American Society of Clinical Oncology (ASCO), the American Cancer Society, and the World Health Organization all recommend providing palliative care interventions simultaneously with treatment for advanced cancer [1013].

Despite the proven benefit of early palliative care involvement, integration of palliative care into standard oncologic treatment for pancreatic cancer is not always straightforward. Referral to a specialized palliative care service can be delayed because of the patients' and physicians' misconception about palliative care being an alternative or non-traditional care strategy [13]. Additionally, there may be geographical barriers, such as inadequate hospice care, in more rural areas [14]. Availability of palliative care services has also become an issue as the field expands faster than providers can be trained and the availability of palliative care services and training varies widely [15-16].

Oncologists and palliative care specialists have a shared responsibility to openly discuss prognosis and recommend end-of-life care options at appropriate times in a cancer patient's disease course. The aim of this study is to assess how frequently were patients with pancreatic cancer referred to palliative care service at our institution with the aim of improving patient outcomes.

The abstract of this research was submitted for the annual ASCO Meeting 2021, and the abstract was published online in the Journal of Clinical Oncology, volume 39, 2021.

\section{Materials And Methods}

We retrospectively reviewed the electronic medical records of all patients diagnosed with pancreatic cancer at Allegheny Health Network from 2009-2020. Patients with significant missing demographic or follow-up data were excluded. Demographic information was collected from admissions face sheets. Clinical staging data is in compliance with the American Joint Committee on Cancer 8th edition staging system [17]. Information on pain was collected from the initial visit history and physical exam, and pain was rated by the patient on a scale of 1-10 with 1 being “minimal pain" and 10 being “the worst pain I've ever felt." Records were reviewed for whether the patient was referred to palliative care services at any time, in the inpatient or outpatient settings, prior to their death or last contact. Summary statistics are presented as absolute numbers and percentages for categorical variables and median with interquartile range for continuous variables. To assess attitudes on palliative care referral within the cancer center, surveys were sent to all hematology and oncology fellows and staff. There was a $40 \%$ response rate for fellows and a $30 \%$ response rate for attending physicians. The study was exempted by the institutional review board of Allegheny Health Network.

\section{Results}

Of the 171 patients reviewed, 121 completed the entire treatment and evaluation within our health network. The baseline characteristics are outlined in Table 1 . The median age was 63 years (IQR 40-91 years); 55 patients (45.5\%) were male; the majority were white (107 patients, $88.4 \%$ ). At the time of diagnosis, $28.1 \%$ of our patients (34 patients) had stage IV disease. Following patients through 2020, 86 patients (71\%) eventually developed stage IV disease, and $19.8 \%$ of those patients with documented stage IV disease had documented palliative care referrals in the outpatient settings provided by oncologists. Eight patients had pain documented at diagnosis, six patients underwent celiac plexus neurolysis, 31 underwent palliative radiation and 43 patients had documented opiate use. 


\section{Cureus}

\section{Characteristic}

Median Age, y

Gender

Male

Female

Race

White

Black

Non-Black Hispanic

Other

Stage at Diagnosis

I

II

III

IV

Patients Reporting Pain at Diagnosis

Patients Listed as Stage IV by 2020

Palliative Referra

No Palliative Referral
Number of patients $(\%)(n=121)$

63.0

$55(45.5 \%)$

$66(54.5 \%)$

$107(88.4 \%)$

$11(9.0 \%)$

$1(0.8 \%)$

$2(1.7 \%)$

$48(39.7 \%)$

$29(24.0 \%)$

$10(8.3 \%)$

$34(28.1 \%)$

$88(72.7 \%)$

$86(71.0 \%)$

24/86 (27.9\%)

$62 / 86(72.1 \%)$

TABLE 1: Baseline characteristics for all patients diagnosed with pancreatic cancer at Allegheny General Hospital from 2009-2020

Palliative interventions and associated pain ratings are shown in Table 2. Of the six patients who underwent celiac plexus neurolysis, only four documented pain at the six-month follow-up. Of the 31 patients who underwent palliative radiation, six documented pain at the six-month follow-up.

\begin{tabular}{|l|l|l|}
\hline Palliative Intervention & Patients with Pain at Diagnosis (No.) & Patients with Pain at 6 Months (No.) \\
\hline Celiac Plexus Neurolysis & 6 & 4 \\
\hline Radiation & 31 & 6
\end{tabular}

TABLE 2: Number of patients who received either celiac plexus neurolysis or radiation with palliative intent as part of treatment for advanced pancreatic cancer

\section{Discussion}

Although the benefits of the early integration of palliative care into the treatment of pancreatic cancer have been demonstrated, the creation of a system whereby providers can be adequately trained in palliative care and sufficient palliative care recourses are available has not yet been achieved. The results of our internal review reflect the need for our program to create a more unified approach to palliative care.

As the support behind the integration of palliative care services into the standard care of patients diagnosed with pancreatic cancer continues to grow, barriers are constantly being identified. We prefer to think of barriers to the successful integration of palliative care as either oncologist-driven, patient-driven, or system-driven. Oncologist-driven factors include inadequate knowledge about palliative care or negative 


\section{Cureus}

attitudes toward palliative care services. A 2012 survey of 603 Canadian medical oncologists, radiation oncologists, and surgical oncologists revealed that $84 \%$ of respondents referred terminally ill patients to palliative care but only $37 \%$ were aware that patients actively receiving chemotherapy could be referred to palliative care services; interestingly, one-third of respondents reported they would have referred to palliative care earlier if it was renamed "supportive care [18].” A 2015 survey of 182 oncologists by Hui et al. again found the name "supportive care" to be preferred and that the name "palliative care" was more likely to be synonymous with "hospice" (PC, 53\% vs. SC, 6\%; < .001) [19]. These findings may suggest that oncologists are uncomfortable with the term "palliative care" and could be less likely to refer because they find it incongruent with active treatment. Another oncologist-driven factor may be inadequate training in the field. A review of 254 second-year oncology fellows in the United States showed that only $26 \%$ had completed a palliative care rotation; when tested on basic palliative care knowledge, the fellows scored a median of 2 of 4 , and only $23 \%$ could correctly perform an opiate conversion [20].

Patients themselves are often resistant to the idea of palliative care. A 2016 trial by Zimmerman et al. randomized patients with advanced cancer who received either standard care or early palliative care and then surveyed both patients and caregivers about attitudes toward palliative care. Initial perceptions in both trial arms about palliative care included associations with death, comfort care, or hopelessness [21]. A common theme was that palliative care was synonymous with death and with care in the last weeks of life [21].

Finally, systems-driven factors include inadequate availability of palliative care resources or lack of infrastructure to support timely access to these resources. A 2010 survey review by Hui et al. showed that while $98 \%$ of National Cancer Institute (NCI)-designated cancer centers had a palliative care program, only $78 \%$ of non-NCI centers had such a program $(\mathrm{P}=0.002)$; NCI centers were also much more likely to have an outpatient palliative care clinic $(59 \%$ vs $22 \%, \mathrm{P}<0.001)[16]$. As NCI-designated cancer centers represent about $4 \%$ of all cancer treatment centers in the United States, there may be many patients who simply do not have access to these resources due to geographical location [22].

To further investigate roadblocks to the successful integration of palliative care within our own program, we performed an internal survey of fellows and facilities to gauge attitudes toward palliative care. Selected survey responses are shown in Figures 1-2. While this survey is not internally validated, we felt it helpful in guiding our evaluation of the integration of palliative care services at our institution. Selected survey responses are shown in Figure 1 . While $100 \%$ of respondents said they had received formal training on palliative care services, only $60 \%$ recognized that palliative care services should be involved at the time of diagnosis of pancreatic cancer. Identified barriers included the unavailability of palliative care services (20\%), palliative care services were offered but declined by the patient or family (40\%), and palliative care services were available but felt to be inappropriate (20\%).
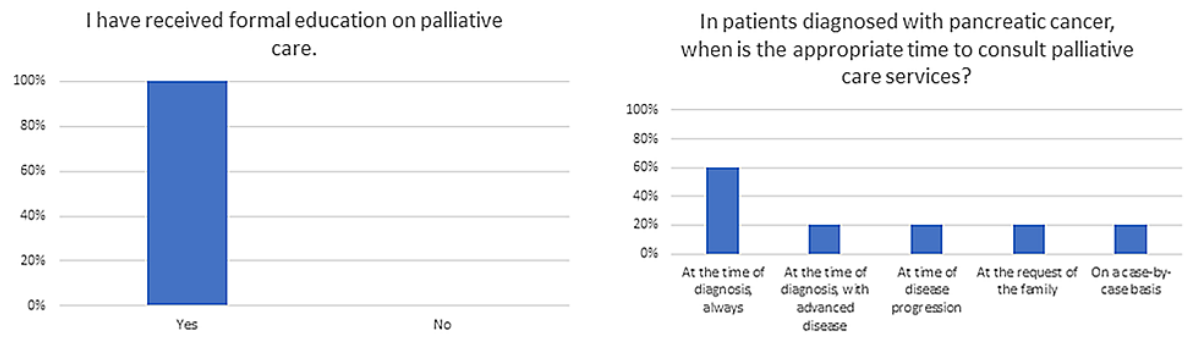

FIGURE 1: Selected survey responses are shown with the question-andanswer choices as they were presented in the survey. Data are presented as a percentage of respondents. 


\section{Cureus}

In patients diagnosed with pancreatic cancer, what has been a barrier to engaging palliative care?

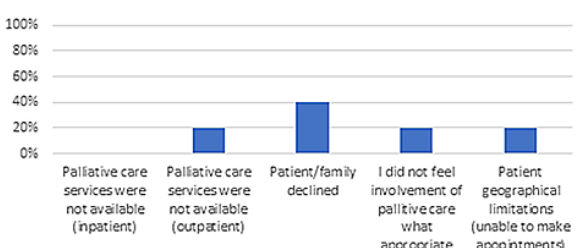

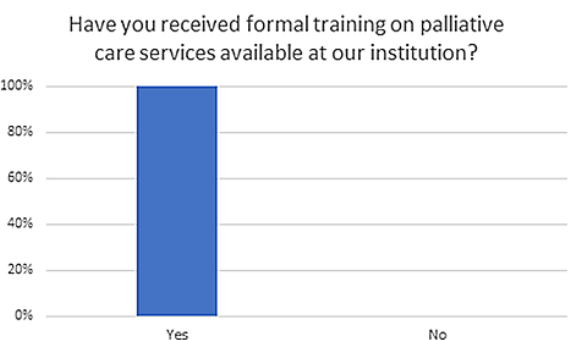

FIGURE 2: Continued: Selected survey responses are shown with the question-and-answer choices as they were presented in the survey. Data are presented as a percentage of respondents.

The limitations of this study include its small sample size. We evaluated 171 patients within one community-based cancer center in the outpatient setting. Our results may be difficult to extrapolate to larger populations or to other hospital systems and cancer treatment centers. We also recognized our results may be open to bias due to missing data, as our records did not have consistently recorded pain ratings with each visit, which can make these results difficult to interpret.

Moving forward, we hope to improve palliative care integration in our patients' care by adding a hard stop to electronic medical records to remind physicians to offer palliative care to our patients with pancreatic cancer at the time of diagnosis. We also plan to arrange medical grand rounds and various lectures to increase awareness of the importance of palliative care in this setting.

\section{Conclusions}

The integration of palliative care is a vital part of usual pancreatic cancer treatment and current treatment guidelines reflect this. In our program, we fall short of providing timely palliative care consultation to our patients diagnosed with pancreatic cancer. An internal survey revealed a lack of oncologist education, patient education, and resource availability as barriers to successful palliative referral. We hope to use this information to further refine our practices and provide adequate palliative care integration to our patients with pancreatic cancer.

\section{Additional Information}

\section{Disclosures}

Human subjects: Consent was obtained or waived by all participants in this study. Institutional review board of Allegheny Health Network issued approval Exempt. Our data set received exempt status from the institutional review board of Allegheny Health Network. Animal subjects: All authors have confirmed that this study did not involve animal subjects or tissue. Conflicts of interest: In compliance with the ICMJE uniform disclosure form, all authors declare the following: Payment/services info: All authors have declared that no financial support was received from any organization for the submitted work. Financial relationships: All authors have declared that they have no financial relationships at present or within the previous three years with any organizations that might have an interest in the submitted work. Other relationships: All authors have declared that there are no other relationships or activities that could appear to have influenced the submitted work.

\section{References}

1. Ilic M, Ilic I: Epidemiology of pancreatic cancer. World J Gastroenterol. 2016, 22:9694-705. 10.3748/wjg.v22.i44.9694

2. Ansari D, Tingstedt B, Andersson B, et al.: Pancreatic cancer: yesterday, today and tomorrow . Future Oncol. 2016, 12:1929-46. 10.2217/fon-2016-0010

3. Del Chiaro M, Rangelova E, Halimi A, et al.: Pancreatectomy with arterial resection is superior to palliation in patients with borderline resectable or locally advanced pancreatic cancer. HPB (Oxford). 2019, 21:219-25. 10.1016/j.hpb.2018.07.017

4. Fiorentini G, Sarti D, Casadei V, et al.: Modulated electro-hyperthermia as palliative treatment for pancreatic cancer: a retrospective observational study on 106 patients. Integr Cancer Ther. 2019, 18:1534735419878505. 10.1177/1534735419878505

5. Axtner J, Steele M, Kröz M, Spahn G, Matthes H, Schad F: Health services research of integrative oncology in palliative care of patients with advanced pancreatic cancer. BMC Cancer. 2016, 16:579. 10.1186/s12885016-2594-5

6. Rabow MW, Petzel MQ, Adkins SH: Symptom management and palliative care in pancreatic cancer . Cancer J. 2017, 23:362-73. 10.1097/PPO.0000000000000293

7. Boyd AD, Brown D, Henrickson C, et al.: Screening for depression, sleep-related disturbances, and anxiety in 
patients with adenocarcinoma of the pancreas: a preliminary study. ScientificWorldJournal. 2012, 2012:650707. 10.1100/2012/650707

8. Clark KL, Loscalzo M, Trask PC, Zabora J, Philip EJ: Psychological distress in patients with pancreatic cancer -an understudied group. Psychooncology. 2010, 19:1313-20. 10.1002/pon.1697

9. Turaga KK, Malafa MP, Jacobsen PB, Schell MJ, Sarr MG: Suicide in patients with pancreatic cancer. Cancer. 2011, 117:642-7. 10.1002/cncr.25428

10. Vanbutsele G, Pardon K, Van Belle S, et al.: Effect of early and systematic integration of palliative care in patients with advanced cancer: a randomised controlled trial. Lancet Oncol. 2018, 19:394-404. 10.1016/S1470-2045(18)30060-3

11. Dose AM, Rhudy LM: Perspectives of newly diagnosed advanced cancer patients receiving dignity therapy during cancer treatment. Support Care Cancer. 2018, 26:187-95. 10.1007/s00520-017-3833-2

12. Hui D, Hannon BL, Zimmermann C, Bruera E: Improving patient and caregiver outcomes in oncology: teambased, timely, and targeted palliative care. CA Cancer J Clin. 2018, 68:356-76. 10.3322/caac.21490

13. Fogel EL, Shahda S, Sandrasegaran K, et al.: A multidisciplinary approach to pancreas cancer in 2016: a review. Am J Gastroenterol. 2017, 112:537-54. 10.1038/ajg.2016.610

14. Charlton M, Schlichting J, Chioreso C, Ward M, Vikas P: Challenges of rural cancer care in the United States . Oncology (Williston Park). 2015, 29:633-40.

15. Hui D, Bruera E: Integrating palliative care into the trajectory of cancer care . Nat Rev Clin Oncol. 2016, 13:159-71. 10.1038/nrclinonc.2015.201

16. Hui D, Elsayem A, De la Cruz M, et al.: Availability and integration of palliative care at US cancer centers . JAMA. 2010, 303:1054-61. 10.1001/jama.2010.258

17. Shi S, Hua J, Liang C, et al.: Proposed modification of the 8th edition of the AJCC Staging System for pancreatic ductal adenocarcinoma. Ann Surg. 2019, 269:944-50. 10.1097/SLA.0000000000002668

18. Wentlandt K, Krzyzanowska MK, Swami N, Rodin GM, Le LW, Zimmermann C: Referral practices of oncologists to specialized palliative care. J Clin Oncol. 2012, 30:4380-6. 10.1200/JCO.2012.44.0248

19. Hui D, Park M, Liu D, Reddy A, Dalal S, Bruera E: Attitudes and beliefs toward supportive and palliative care referral among hematologic and solid tumor oncology specialists. Oncologist. 2015, 20:1326-32. 10.1634/theoncologist.2015-0240

20. Buss MK, Lessen DS, Sullivan AM, Von Roenn J, Arnold RM, Block SD: Hematology/oncology fellows' training in palliative care: results of a national survey. Cancer. 2011, 117:4304-11. 10.1002/cncr.25952

21. Zimmermann C, Swami N, Krzyzanowska M, et al.: Perceptions of palliative care among patients with advanced cancer and their caregivers. CMAJ. 2016, 188:E217-27. 10.1503/cmaj.151171

22. NIH. National Cancer Institute. NCI-designated cancer centers . (2019). Accessed: May 17, 2021: https://www.cancer.gov/research/infrastructure/cancer-centers. 